\title{
THE UNIQUENESS CLASS FOR THE CAUCHY PROBLEM FOR PSEUDOPARABOLIC EQUATIONS
}

\author{
WILLIAM RUNDELL
}

\begin{abstract}
It is shown that the class of functions satisfying $|u(x, t)|<$ $M e^{\alpha|x|}$ forms a uniqueness class for the Cauchy problem for pseudoparabolic equations. The surprising fact is that, unlike the case of parabolic equations, the constant $\alpha$ is not arbitrary but depends on the coefficients of the equation.
\end{abstract}

Introduction. This paper is concerned with determining the uniqueness class for the Cauchy problem for the pseudoparabolic equation

$$
L u-M u_{t}-c(x) u_{t}+M u=0 .
$$

Here $M$ is an elliptic partial differential operator of second order and $c(x)$ is a positive coefficient. All coefficients are assumed to be bounded.

Since solutions of (1.1) are closely related to solutions of the associated parabolic equation

$$
P u=c(x) u_{t}-M u=0
$$

(cf. [1], [3]), one would expect a similarity in the uniqueness class for the Cauchy problem, that is a solution that takes prescribed values on the axis $t=0$.

For the parabolic equation if $|u(x, t)|<C e^{\alpha x^{2}}$ for arbitrary fixed $\alpha$, then there is a unique solution for the Cauchy problem. For equation (1.1) we shall see that the uniqueness class consists of functions of first order growth, that is, $|u(x, t)|<c e^{\alpha|x|}$. The surprising fact is that $\alpha$ in this case is no longer arbitrary but depends on the operator $L$. More specifically it depends on the lower bound for the coefficient $c(x)$ and the modulus of ellipticity of the operator $M$. We shall illustrate this with an example, deferring the statement of the main theorem until the next section.

EXAMPLE. There is a nontrivial solution to the Cauchy problem,

$$
\begin{aligned}
u_{x x t}-u_{t}+u_{x x} & =0, \\
u(x, 0) & =0,
\end{aligned}
$$

that satisfies the estimate $|u(x, t)|<e^{\sqrt{2}|x|}$.

We let

$$
u(x, t)=\sum_{n=0}^{\infty} \frac{x^{2 n}}{(2 n) !} a_{n}(t)
$$

Received by the editors September 11, 1978.

AMS (MOS) subject classifications (1970). Primary 35G10, 35 Q99. 
where

$$
\begin{aligned}
a_{n}^{\prime}(t)-a_{n-1}^{\prime}(t) & =a_{n}(t), \quad n=0,1,2, \ldots, \\
a_{n}(0) & =0, \quad n=0,1,2, \ldots
\end{aligned}
$$

Under these conditions it is easily verified that $u(x, t)$ is a solution to (1.3) and (1.4). We choose $a_{0}(t)$ to satisfy $0<a_{0}(t)<1$.

Equations (1.6) become

$$
a_{n}(t)=a_{n-1}(t)-\int_{0}^{t} e^{-(t-\tau)} a_{n-1}(\tau) d \tau
$$

that is

$$
a_{n}(t)=(I-T)^{n} a_{0}(t)
$$

where

$$
T f=\int_{0}^{t} e^{-(t-\tau)} f(\tau) d \tau
$$

Thus

$$
a_{n}(t)=\sum_{r=0}^{n}\left(\begin{array}{l}
n \\
r
\end{array}\right)(-1)^{r} T^{r} a_{0}
$$

where

$$
T^{r} a_{0}=\frac{1}{(r-1) !} \int_{0}^{t}(t-\tau)^{r-1} e^{-(t-\tau)} a_{0}(\tau) d \tau
$$

Thus

$$
\left|T^{r} a_{0}(t)\right|<1, \quad r=1,2, \ldots, \quad t>0 .
$$

The last estimate follows by taking the maximum of the integrand and using Stirling's formula. Thus $a_{n}(t)$ satisfies

$$
a_{n}(t)<2^{n}, \quad n=0,1, \ldots, \quad t>0,
$$

and equation (1.5) yields the estimate

$$
|u(x, t)|<e^{\sqrt{2}|x|} .
$$

We first introduce some notation. We denote by $D_{R}$ the ball $\left\{x \in \mathbf{R}^{n}\right.$ : $|x|<R\}$ and by $C^{k+\alpha}\left(D_{R}\right)$ the Banach space consisting of those functions whose derivatives of order $k$ are Hölder continuous in $D_{R}$ with exponent $\alpha$, $0<\alpha<1$. It is assumed that this space is equipped with its usual norm. By $C_{0}^{k+\alpha}\left(D_{R}\right)$ we mean the closed subset of $C^{k+\alpha}\left(D_{R}\right)$ consisting of those functions that vanish on the sphere $|x|=R$.

We shall consider solutions $u(x, t)$ of the equation

$$
L u=(M-c I) u_{t}+M u=0
$$

for $x \in \mathbf{R}^{n}, t>0$. Here $I$ is the identity map and $M$ is an elliptic partial differential operator of the form 


$$
M=-\sum_{i, j=1}^{n} m_{i j}(x) \frac{\partial^{2}}{\partial x_{i} \partial x_{j}}+\sum_{i=1}^{n} m_{i}(x) \frac{\partial}{\partial x_{i}}+m(x)
$$

such that

(a) the coefficients lie in $C^{\alpha}\left(D_{R}\right)$ for each $R>0$ and there is a constant $K$ such that $\left|m_{i j}(x)\right|<K,\left|m_{i}(x)\right|<K, 0<m(x)<K, x \in \mathbf{R}^{n}, 1<i, j<n$;

(b) there is a constant $m_{0}>0$ such that

$$
\sum_{i, j=1}^{n} m_{i j}(x) \xi_{i} \xi_{j}>m_{0} \sum_{i=1}^{n} \xi_{i}^{2}
$$

whenever $x$ and $\xi=\left(\xi_{1}, \ldots, \xi_{n}\right) \in \mathbf{R}^{n}$.

We shall also assume that the coefficient $c(x)$ lies in $C^{\alpha}\left(D_{R}\right)$ for all $R>0$ and, for some positive constants $\mu$ and $K$,

$$
0<\mu<c(x)<K, \quad x \in \mathbf{R}^{n} .
$$

By a solution to (2.1) we mean a function $u(x, t)$ such that for each $R>0$ : (i) $u(\cdot, t) \in C^{2+\alpha}\left(D_{R}\right)$ for all $t>0$, (ii) the map from $[0, \infty)$ to $C^{2+\alpha}\left(D_{R}\right)$, $t \rightarrow u(\cdot, t)$ is continuously differentiable, (iii) $u(x, t)$ satisfies (2.1) for $x \in \mathbf{R}^{n}$ and $t>0$.

Our main result is the following:

TheOREM. Let $u(x, t)$ be a solution of $(2.1)$ with $u(x, 0)=0$ that satisfies the estimate

$$
|u(x, t)|<C e^{\alpha|x|}, \quad x \in \mathbf{R}^{n}, \quad t>0,
$$

for some constants $C$ and $\alpha$ with $\alpha<\sqrt{\mu / m_{0}}$. Then $u(x, t)=0$ for $(x, t) \in$ $\mathbf{R}^{n} \times[0, \infty)$.

Proof. Fix $R>0$, and let $\phi_{\lambda}(x)$ satisfy

$$
\begin{aligned}
M \phi_{\lambda} & =\lambda^{2} m_{0} \phi_{\lambda}, \quad 0<\lambda<\sqrt{\mu / m_{0}}, \quad|x|<R, \\
\phi_{\lambda} & =e^{\lambda R}, \quad|x|=R .
\end{aligned}
$$

Then an application of the maximum principle for elliptic operators shows that $\phi_{\lambda}(x)>0$ for $x \in D_{R}$. The presence of the constant $m_{0}$ in (2.4) means that we have essentially divided equation (2.1) by $m_{0}$ in order to "normalize" the operator $M$.

If we put

$$
\psi(x, t)=C e^{(\alpha-\lambda) R} e^{\lambda^{2} m_{0} t\left(\mu-\lambda^{2} m_{0}\right)^{-1}} \phi_{\lambda}(x)
$$

then

$$
v=\psi-u
$$

satisfies

$$
\begin{array}{rlrl}
L v & =f(x, t), & x \in D_{R}, \quad t>0, \\
v(x, 0) & =h(x), \quad x \in D_{R}, \\
v(x, t) & =g(t), \quad|x|=R, \quad t>0,
\end{array}
$$


where

$$
\begin{aligned}
f(x, t) & =C e^{(\alpha-\lambda) R} e^{\lambda^{2} m_{0} t\left(\mu-\lambda^{2} m_{0}\right)}\left\{\frac{\lambda^{2} m_{0}(\mu-c(x))}{\mu-\lambda^{2} m_{0}}\right\} \phi_{\lambda}(x)<0, \\
h(x) & =C e^{(\alpha-\lambda) R} \phi_{\lambda}(x) \geqslant 0, \\
g(t) & >C e^{\alpha R} e^{\lambda^{2} m_{0} t\left(\mu-\lambda^{2} m_{0}\right)^{-1}}-C e^{\alpha R} \geqslant 0 .
\end{aligned}
$$

We wish to show that $v(x, t) \geq 0$ for $x \in D_{R}, t>0$. Unlike the case of a parabolic equation we are unable to achieve this by appealing directly to a maximum principle (cf. [2]). We shall instead convert the initial boundary value problem (2.7) into an integral equation with positive kernel and free term.

We define $G(x, t) \in C^{2+\alpha}\left(D_{R}\right)$ by

$$
\begin{aligned}
(M-c(x) I) G & =0, \quad|x|<R, \quad t>0, \\
G(x, t) & =e^{t} g(t), \quad|x|=R, \quad t>0 .
\end{aligned}
$$

Again by the maximum principle for elliptic operators we have that $G>0$ in $D_{R} \times[0, \infty)$. As a function of $t, G(\cdot, t)$ is continuously differentiable for $t>0$. If we make the transformation

$$
w(x, t)=e^{t} v(x, t)-G(x, t),
$$

then $w(x, t) \in C_{0}^{2+\alpha}\left(D_{R}\right), w(\cdot, t)$ is continuously differentiable in $t$ and satisfies

$$
\begin{aligned}
(M-c(x) I) w_{t}+c(x) w & =e^{t} f-G, \quad|x|<R, \quad t>0, \\
w(x, 0) & =h(x)-G(x, 0), \quad|x|<R, \\
w(x, t) & =0, \quad|x|=R, \quad t>0 .
\end{aligned}
$$

If we rewrite this in the space $C\left(D_{R}\right)$ we obtain

$$
\begin{aligned}
w_{t}-A w & =B\left(e^{t} f-G\right), \\
w(0) & =h-G(\cdot, 0),
\end{aligned}
$$

where $A$ and $B$ denote the operators from $C^{\alpha}\left(D_{R}\right)$ to $C_{0}^{2+\alpha}\left(D_{R}\right)$ defined by

$$
\begin{aligned}
& A u=-(M-c(x) I)^{-1} c(x) u, \\
& B u=-(M-c(x) I)^{-1} u .
\end{aligned}
$$

The maximum principle shows that $u>0$ pointwise in $D_{R}$ implies that $A u>0$ and $B u>0$ pointwise.

If we integrate (2.14) with respect to $t$ from 0 to $t$ we obtain

$$
w(t)=\int_{0}^{t} A w d \tau+H(t)
$$

where

$$
H(t)=\int_{0}^{t} B\left(G-e^{\tau} f\right) d \tau+h-G(, 0)
$$


We note that the integrand in (2.18) is nonnegative and hence $H(t)$ will be nonnegative provided $h(x)-G(x, 0)>0$ in $D_{R}$. However the function $\theta(x)$ $=h(x)-G(x, 0) \in C_{0}^{2+\alpha}\left(D_{R}\right)$ satisfies

$$
(M-c(x) I) \theta(x)=C e^{(\alpha-\lambda) R}\left[\lambda^{2} m_{0}-c(x)\right] \phi_{\lambda}(x)<0
$$

since $\lambda^{2} m_{0}<\mu<c(x)$ for $x \in D_{R}$ and $\phi_{\lambda}(x)>0$ in $D_{R}$. The maximum principle now implies that $\theta(x)>0$.

Picard iteration applied to (2.17) shows that $w(x, t)>0$ for $x \in D_{R}, t>0$. The positivity of $G$ and (2.12) yields the desired conclusion that $v(x, t)>0$ in $D_{R} \times[0, \infty)$.

Thus for each $R>0$

$$
u(x, t)<\psi(x, t)=C e^{(\alpha-\lambda) R} e^{\lambda^{2} m_{0} t\left(\mu-\lambda^{2} m_{0}\right)^{-1}} \phi_{\lambda}(x) .
$$

For any $\alpha<\sqrt{\mu / m_{0}}$ we may choose a $\lambda$ such that $\alpha<\lambda<\sqrt{\mu / m_{0}}$ and let $R \rightarrow \infty$ in (2.20) to obtain $u(x, t)<0$ in $D_{R} \times[0, \infty)$. Applying the above analysis to $-u$ yields the conclusion of the theorem.

\section{REFERENCES}

1. R. W. Carroll and R. E. Showalter, Singular and degenerate Cauchy problems, Academic Press, New York, 1976.

2. W. Rundell and M. Stecher, The nonpositivity of solutions to pseudoparabolic equations, Proc. Amer. Math. Soc.75 (1979), 251-254.

3. T. W. Ting, Parabolic and pseudoparabolic differential equations, J. Math. Soc. Japan 21 (1969), 440-453.

Department of Mathematics, Texas AdM University, College Station, Texas 77843 